\title{
Beneficios de la clinoptilolita colombiana en la germinación de maíz (Zea mays L.) en suelo arcilloso
}

\author{
Corzo Díaz Andrea Carolina', Noriega Hernández Aristides José , Alvarado Ibarra Juana ${ }^{2, *}$
}

1 Fundación Universitaria del Área Andina

2 Departamento de Investigación en Polímeros y Materiales, Universidad de Sonora

\begin{abstract}
Resumen
En este ensayo se utilizó una zeolita natural de origen colombiano tipo "clinoptilolita" y un fertilizante triple 15 (NPK) con el objetivo de mejorar las propiedades de un suelo arcilloso y mejorar la germinación de maíz. Mediante un experimento no aleatorio se hizo una prueba de viabilidad con treinta semillas de maíz. De manera simultánea, se realizaron diez experimentos por triplicado, variando la cantidad de suelo y la relación de zeolita-fertilizante. En la prueba de viabilidad se encontró que a las 120 h se tiene una tasa de germinación del $80 \%$. En los experimentos se observó que la zeolita mejora la capacidad de filtrado y reduce la compactación del suelo. Se concluyó que la mezcla de zeolita y fertilizante disminuyen el tiempo de germinado del maíz, el uso de agua de riego y la cantidad de fertilizante a usar en el cultivo. Por lo anterior, este trabajo es una alternativa con potencial para contribuir con el objetivo del desarrollo sustentable 15, sobre la vida de ecosistemas terrestres al tener la posibilidad de recuperar suelos infértiles.
\end{abstract}

Palabras Clave: Objetivos del desarrollo sustentable, Zeolita, Fertilidad del suelo.

\section{Benefits from colombian clinoptilolite in maize seeds (Zea mays L.) germination on clay soil}

\begin{abstract}
In this trial, a natural zeolite of Colombian origin type "clinoptilolite" and a triple 15 fertilizer (NPK) were used with the aim of improving the properties of a clay soil and improving maize germination. A non-random experiment did a feasibility test with thirty maize seeds. Simultaneously, ten experiments were conducted per triplicate, varying the amount of soil and the ratio of zeolite-fertilizer. The feasibility test found that at 120 $\mathrm{h}$ there is a germination rate of $80 \%$. Experiments found that zeolite improves filtering capacity and reduces soil compaction. We concluded that the mixture of zeolite and fertilizer reduces the germination time of maize seeds, the use of irrigation water and the amount of fertilizer to be used in the crop. Therefore, this work is an alternative to contribute to the objective of sustainable development 15, on the life of terrestrial ecosystems by having the possibility of recovering infertile soils.
\end{abstract}

Keywords: Sustainable development goals, Zeolite, Soil fertility. 


\section{INTRODUCCION}

Un suelo sano proporciona innumerables recursos y servicios ambientales, aunado a que es la base de la producción de alimentos. Sin embargo, el recurso suelo se está deteriorando, se están modificando sus usos e incrementado la deforestación (FAO, 2015). Por lo anterior, cerca de 200 países del mundo están trabajando en el compromiso que asumieron con los 17 Objetivos del Desarrollo Sustentable (ODS) para lograr al 2030 la erradicación de la pobreza extrema, combatir la desigualdad y la injusticia y proteger el ambiente (FAO, 2020). Con lo anterior se busca garantizar la permanencia del ser humano en el planeta y que las futuras generaciones cuenten con las condiciones óptimas para vivir en el planeta (https://www.campusfad.org/viralizaods/que-son-los-ods/).

Es sabido el poder de amortiguación que el suelo tiene para inactivar los efectos negativos causados por el proceso de la agricultura, sin embargo, cuando un suelo ha sido superado en su capacidad de amortiguación, pasa a ser un problema en lugar de mantenerse como un factor de protección, ya que se modifican sus equilibrios biogeoquímicos y con ello las propiedades físicas, químicas y biológicas del suelo (Quichimbo, et al., 2012). Los suelos arcillosos se caracterizan por tener poca materia orgánica y biodiversidad, por lo que la producción de materia orgánica, la transpiración de las plantas y el rendimiento en la biomasa se reducen significativamente, aun haya variantes como la especie de la planta y el manejo de riego afectando directamente el crecimiento de la planta, la estabilidad estructural del suelo, su tasa de infiltración y su capacidad de retención de agua (Katerji, et al., 2000).

Se han reportado varias investigaciones que presentan métodos para mejorar los suelos y con ello garantizar la seguridad alimentaria, estos métodos buscan mejorar el contenido de materia orgánica, la actividad microbiana, la absorción del agua, la conductividad hidráulica saturada, la estabilidad estructural, la densidad aparente del suelo y la porosidad de éste (Sameen, et al., 2016). La fertilidad del suelo está relacionada con la capacidad que tiene para ser el hábitat de animales, micro y macroorganismos, todos interconectados entre sí, ya que es un sistema diverso y complejo (CIMMYT, 2013). Sin embargo, para sustentar la vida vegetal, se dependerá de la disponibilidad de nutrientes y del espacio físico para el crecimiento de las raíces y el movimiento de gases (Céspedes, 2010).

Las zeolitas son aluminosilicatos cristalinos y porosos, lo que permite el intercambio iónico sin cambiar su estructura cristalina, por lo que son una alternativa de solución en problemas de suelo, siendo así del interés de los científicos para su uso en la agricultura (López, et al., 2010). Las zeolitas, proveen de nutrientes necesarios para los cultivos, mejoran la capacidad de hidratación y deshidratación de los suelos, permite el intercambio del amonio por sodio y potasio, disminuye la aplicación de fertilizantes y reduce las pérdidas por volatilización y lixiviación de éstos (Zahedi, et al., 2012). La zeolita no actúa como fertilizante, pero permite recuperar su eficiencia y disponibilidad controlada de los cationes que las plantas utilizan (Costafreda, 2011).

En Colombia predomina el cultivo tradicional practicado en pequeñas extensiones de tierra y dirigido al autoconsumo. El proceso se realiza en suelos poco fértiles, no se hace uso de semillas certificadas, ni de agroquímicos o de mecanización, lo que genera rendimientos de cosecha muy bajos (Grande y Orozco, 2013). Por lo anterior, el objetivo de esta investigación fue evaluar la utilización de una zeolita natural tipo clinoptilolita y un fertilizante NPK triple 15 para mejorar las propiedades de un suelo arcilloso doméstico y reducir el tiempo en la germinación de la semilla de maíz al propiciar las condiciones de humedad y disponibilidad de nutrientes.

\section{MATERIALES Y MÉTODOS}

\section{Lugar de estudio}

El estudio se llevó a cabo en el mes de julio de 2020. Se desarrolló en una residencia ubicada en Colombia, en Cuatro vientos, corregimiento del municipio de El Paso, el cual se encuentra al centro-occidente del Departamento del Cesar. Éste se encuentra a $130 \mathrm{~km}$ de Valledupar, capital del Departamento del Cesar, con una altura de $35.5 \mathrm{msnm}$, y temperaturas que oscilan entre $27^{\circ} \mathrm{C}$ a $32{ }^{\circ} \mathrm{C}$ (GOV.CO, 2020).

\section{Preparación de muestras}

El suelo en estudio corresponde al de una residencia ubicada en la periferia del corregimiento. Se seleccionó un área despejada y sin alteraciones irregulares. Se tomó un área de $30 \mathrm{~cm} \times 30 \mathrm{~cm}$, se escarbó a una profundidad de $10 \mathrm{~cm}$ para obtener $7.605 \mathrm{~kg}$ de suelo, mismo que se colocó en una charola y se puso a secar a la intemperie. Posteriormente, se tamizó la muestra, obteniendo un peso de $4.95 \mathrm{~kg}$ con tamaño de partícula inferior a $3 \mathrm{~mm}$.

La zeolita tipo clinoptilolita de origen natural, fue proporcionada por la empresa ZEOCOL (Zeolitas de Colombia, Pereira Colombia), cuya composición se presenta en la tabla 1. La zeolita se ocupó tal y como la proporcionó la empresa, solo se realizó un secado previo. Se pesó 170 g del material, se colocó en una charola y se secó por $12 \mathrm{~h}$ a la intemperie, teniendo un promedio de temperatura de $32{ }^{\circ} \mathrm{C}$. El porcentaje de humedad se determinó usando la fórmula (CIMMYT, 2013). 
Tabla 1. Composición de la clinoptilolita colombiana

\begin{tabular}{|c|c|c|}
\hline Elemento & $\%$ & Método analítico \\
\hline K & $0.15-3.17$ & NTC 202 Emisión \\
\hline $\mathrm{Ca}$ & $1.5-2.75$ & $\begin{array}{c}\text { NTC } 1369 \\
\text { Absorción Atómica }\end{array}$ \\
\hline$M g$ & $0.5-0.83$ & $\begin{array}{c}\text { NTC } 1369 \\
\text { Absorción Atómica }\end{array}$ \\
\hline $\mathrm{Fe}$ & $1-2.0$ & $\begin{array}{c}\text { NTC } \\
\text { Absorción Atómica }\end{array}$ \\
\hline $\mathrm{Al}$ & $1-15.8$ & LBC 89 Colorimetría \\
\hline $\mathrm{Na}$ & $1.02-1.16$ & NTC 1146 Emisión \\
\hline $\mathrm{Si}$ & $63.13-78.2$ & LBC 89 Colorimetría \\
\hline Parámetro & Unidad & \\
\hline $\mathrm{pH}$ & 7.5 & $\begin{array}{c}\text { NTC } 5167 \\
\text { Potenciométrico }\end{array}$ \\
\hline Humedad & $0.3-5.0 \%$ & NTC 35 Gravimetría \\
\hline Retención de humedad & $30-45 \%$ & NTC5167 Gravimetría \\
\hline $\mathrm{CIC}$ & $50-89 \mathrm{me} / 100 \mathrm{~g}$ & NTC 5167 Volumetría \\
\hline Clinoptilolita & $43-69 \%$ & DRX \\
\hline
\end{tabular}

Fuente: Ficha técnica proporcionada por la empresa ZEOCOL.

$$
h=\frac{P h-P S}{P S} \times 100
$$

Donde; $h$ es el porcentaje de humedad. Ph es el peso de la muestra húmeda y Ps es el peso de la muestra seca.

Se utilizaron 60 semillas de maíz comercial, seleccionadas al azar con buena apariencia a la vista, que no presentaran quiebres, deformaciones o coloraciones atípicas.

\section{Análisis del suelo}

Para determinar la proporción de las partículas (arcilla, limo y arena) de la muestra de suelo, se realizó una prueba por duplicado en la que se colocaron en un recipiente cilíndrico 50 gr del suelo preparado y se adicionó $150 \mathrm{~mL}$ de agua corriente, se agitó por un minuto y se dejó reposar por $24 \mathrm{~h}$. Se midió la altura de cada material sedimentado y se utilizó la fórmula para determinar en cada capa el porcentaje de partículas (http://www.elpaso-cesar.gov.co/municipio/ nuestro-municipio).

$$
F=\frac{E m \times 100}{M}
$$

Donde; F es el porcentaje de partículas, Em es la altura del material sedimentado en $\mathrm{cm}$ y $\mathrm{M}$ es la altura total del suelo en $\mathrm{cm}$. La textura se determinó por el método del triángulo textural y por la tabla granulométrica de dimensión de partícula elemental, ambas establecidas por el Departamento de Agricultura de Estados Unidos (USDA, por sus siglas en inglés).

\section{Prueba de viabilidad}

La prueba de viabilidad de germinación de la semilla se realizó con 30 ensayos. Se tomó una semilla y se cubrió con papel absorbente (CIMMYT, 2013), dejándola en un vaso de germinación y aplicándole agua hasta que el papel estuvo completamente húmedo. Durante 5 días y cada 6 horas se humedeció el papel con $4 \mathrm{~mL}$ de agua, evitando encharcamientos y el desarrollo de hongos. La tasa de germinación de las semillas se verificó con el desarrollo de las plántulas, en cuanto al tamaño y longitud de las radículas principales y secundarias, longitud del coleóptilo y presencia de hojas.

\section{La determinación de viabilidad se realizó con la fórmula}

$$
N=\frac{G \times 100}{S}
$$

Donde; $\mathrm{N}$ es el porcentaje de viabilidad del lote de las semillas, $\mathrm{G}$ el número de semillas germinadas y $\mathrm{S}$ el número total de semillas.

\section{Diseño Experimental}

Se utilizaron 30 macetas de plástico de $88.7 \mathrm{~mL}$ de capacidad, identificando un testigo y nueve tratamientos por triplicado, tal y como se muestra en la tabla 2. En cada maceta se agregó suelo, zeolita tipo clinoptilolita, fertilizante triple 15 NPK en diferentes porcentajes y una semilla de maíz. Se monitoreo el comportamiento de los ensayos durante tres semanas y se regaron según necesidades del ensayo.

Tabla 2. Distribución de porcentaje de materiales para los ensayos

\begin{tabular}{|cccc|} 
Tratamiento & Suelo & Zeolita & Fertilizante \\
\hline A & 100 & $/$ & $/$ \\
\hline B & 90 & 10 & $/$ \\
\hline C & 90 & $/$ & 10 \\
\hline D & 70 & 15 & 15 \\
\hline E & 65 & 25 & 10 \\
\hline F & 50 & 35 & 15 \\
\hline G & 50 & 15 & 35 \\
\hline H & 60 & 20 & 20 \\
\hline I & 60 & 30 & 10 \\
\hline J & 60 & 10 & 30 \\
\hline
\end{tabular}

Fuente: elaboración propia 


\section{RESULTADOS Y DISCUSIÓN}

El suelo presentó una composición de $25.49 \%$ de arena, $31.37 \%$ de limo y $43.13 \%$ de arcilla. Con base en la tabla de clasificación textural, el suelo en estudio es clasificado como arcilloso.

La zeolita presentó una humedad del 3.03\%, valor dentro del intervalo establecido en la ficha técnica del material proporcionada por la empresa ZEOCOL.

Respecto a la prueba de viabilidad, se encontró que a las 48 h se obtuvo una germinación en el $70 \%$ de las semillas en evaluación, presentando radículas. A las 72 h hubo aparición de raíces secundarias y del coleóptilo. A las 120 h la tasa de germinación fue del $80 \%$, lo que indica que las semillas lograron llevar a cabo las tres fases del proceso de germinación, iniciando con la imbibición, seguido por la síntesis de proteínas y finalmente la fase de crecimiento (Besnier, 1989). El coleóptilo presentó una longitud promedio de $1.33 \mathrm{~cm}$, lo que permitirá que la planta crezca adecuadamente (CIMMYT, 2013). Las raíces primarias presentaron una longitud promedio de $5.6 \mathrm{~cm}$ y las secundarias de $3.8 \mathrm{~cm}$. Finalmente, a los cinco días de experimentación, se observó la aparición de segundas hojas, mismas que alcanzaron un tamaño promedio de $15 \mathrm{~cm}$.

En la figura 1 se muestra un comparativo entre la muestra testigo $A$ y la muestra $E$, que contiene $65 \%$ de suelo $-25 \%$ de zeolita $-10 \%$ de fertilizante. Se hace evidente el aporte de la zeolita en la capacidad de drenado, evitando

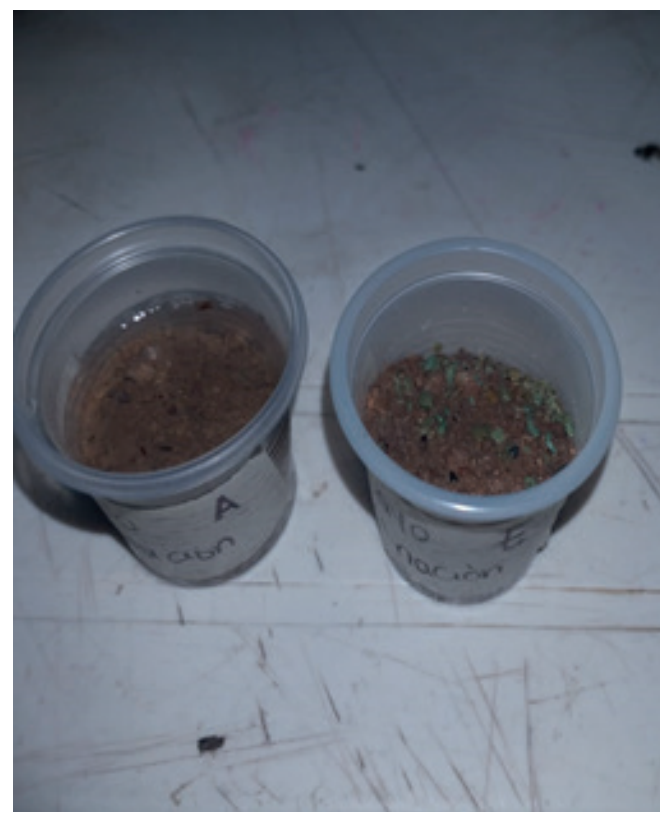

Figura 1. Aporte de la zeolita (lado derecho) en el drenado encharcamientos y disminuyendo la posibilidad de aparición de hongos que enfermen el germinado (CIMMYT, 2013). Se observó que los experimentos con zeolita mantienen húmedo al suelo, disminuyendo la frecuencia de regado. También se pudo apreciar un cambio en la compactación en los experimentos con zeolita. El suelo sin zeolita se compactó mayormente en comparación con los ensayos con zeolita, lo anterior tiene implicación directa en la distribución de oxígeno y en la uniformidad de la humedad (Céspedes, 2010). A las 24 h, las muestras sin zeolita presentaban sequedad del suelo, mientras que las muestras con zeolita se mantenían húmedas, lo que implicó menos uso de agua.

En la fotografía 2, se muestra el tratamiento $F$, compuesto por $50 \%$ de suelo, $35 \%$ de zeolita y $15 \%$ de fertilizante triple 15 , a las 48 h se observó claramente la fase I del proceso de germinación, al darse el inició de la entrada de agua en la semilla y presentarse una absorción rápida y el hinchamiento de la semilla, incrementando su tamaño. El ensayo requirió un volumen de agua de $32 \mathrm{~mL}$, dato que coincide con los estudios de Sousa y colaboradores (2006) quienes mostraron que durante la imbibición hay un aumento de peso; mientras que Méndez y colaboradores (2008) observaron el patrón de absorción rápida. En la fase II se da la activación del metabolismo, síntesis de proteínas y movilización de sustancias como azúcares simples, celulosa y aminoácidos que son transportados y oxidados para suplir el crecimiento (Besnier, 1989). En la fotografía 2 se aprecia el crecimiento del coleóptilo, ocasionado por la entrada de agua con los solutos de la clinoptilolita y el fertilizante disueltos en

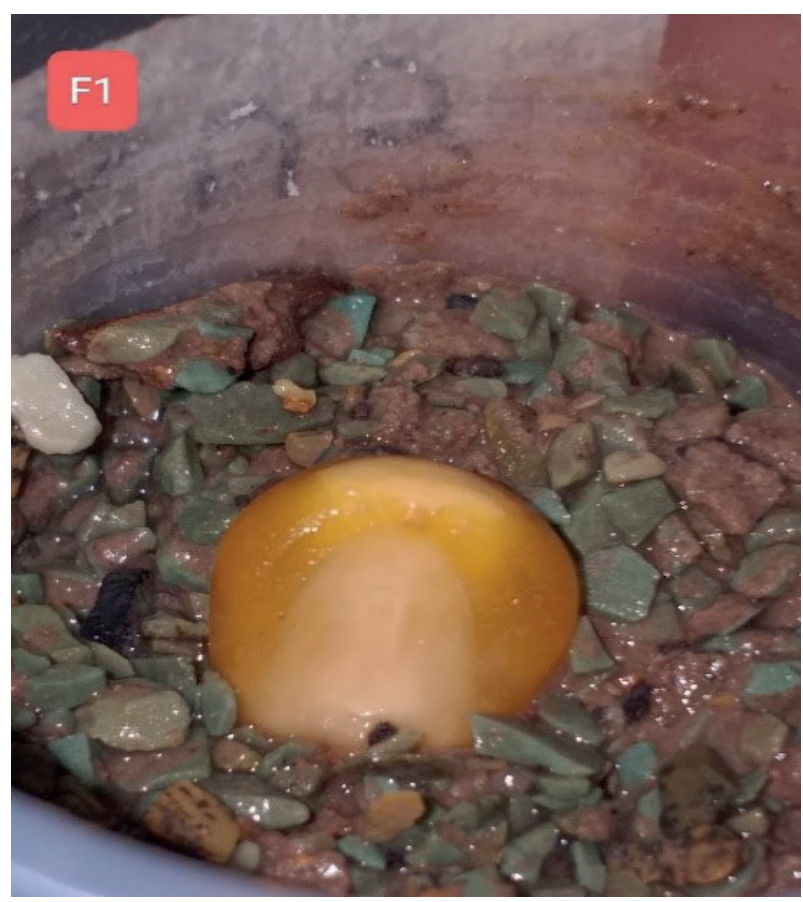

Figura 2. Cambios físicos en la semilla de maíz, se aprecia el crecimiento del coleóptilo 
la solución del suelo, datos similares reportaron SánchezPérez et al (2010). La fase III, que se presenta cuando ocurre la ruptura de la testa y la germinación, no ocurrió, debido a que el ensayo terminó antes de presentarse el evento.

No se aprecian cambios significativos en el desarrollo de la semilla de maíz en los diferentes ensayos con zeolita. El cambio comparativo evidente fue en el ensayo sin zeolita, ya que la semilla de maíz, en los ensayos con zeolita, logra incrementar su tamaño después de $72 \mathrm{~h}$. Es decir, la fase I de la germinación es observable antes en las semillas con zeolita y fertilizante que en las muestras que no los contenían.

\section{CONCLUSIONES}

La zeolita tipo clinoptilolita de Colombia favoreció significativamente las propiedades del suelo en cuanto a la textura, la permeabilidad y la compactación. Los experimentos con cambios distintivos en el suelo y en la semilla fueron los ensayos con zeolita y fertilizante. Se redujo el tiempo de aparición de la fase I de la germinación de maíz en suelos arcillosos al combinar zeolita y fertilizante. Los experimentos con mezclas de zeolita y fertilizante usaron menos agua para el riego que los experimentos sin la mezcla zeolita-fertilizante.

Agradecimientos. Se agradece al programa Delfín por realizar la estancia virtual del XXV Verano de la Investigación Científica y Tecnológica del Pacífico.

\section{BIBLIOGRAFÍA}

Besnier, F. 1989. Semillas, biología y tecnología. MundiPrensa. Madrid.

Céspedes, C. 2010. Manejo de la fertilidad del suelo. C. 4 en Manual de Producción de Frambuesa Orgánica. M.I. González y M.C. Céspedes (Ed.), p. 21-31. Instituto de Investigaciones Agropecuarias. Chillán, Chile.

CIMMYT. 2013. Contenido de humedad del suelo, Guía útil para comparar las prácticas de manejo de cultivo. Rep. CIMMYT. Centro Internacional de Mejoramiento de Maíz y Trigo MasAgro. México.

Costafreda, J.L. 2011. Tectosilicatos con características especiales: las zeolitas naturales. Fundación Gómez Pardo. Madrid.
FAO. 2015. Suelos sanos para una vida sana. Rep. en ONU para la Alimentación y la Agricultura. Roma, Italia.

FAO. 2020. Gestionar sosteniblemente los bosques, luchar contra la desertificación, detener e invertir la degradación de las tierras y detener la pérdida de biodiversidad. Rep. en FAO. oficina de América Latina y el Caribe.

Globe. 2005. Comunicación personal. Protocolo de distribución de partículas del suelo por tamaño.

Grande, C.D. y Orozco, B.S. 2013. Producción y procesamiento del maíz en Colombia. Rev. Guillermo de Ockham. 11(1): 97-110.

Katerji, N., Van Hoorn, J.W., Hamdy, A. y Mastrorilli, M. 2000. Salt tolerance classification of crops according to soil salinity and to water stress day index. Agricultural Water Mangement. 43(1): 99-109.

López, M., Hernández, C., Barahona, M., Martínez, R., Portillo, R. y Rojas, F. 2010. Propiedades fisicoquímicas de la clinoptilolita tratada con fertilizantes a usar como aditivo en el cultivo de Pleurotus ostreatus. Terra Latinoamericana. 28: 1-8.

Méndez, J.R., Merazo, J.F. y Montaño, N.J. 2008. Relación entre la tasa de imbibición y el porcentaje de germinación en semillas de maiz (Zea mays L.), carota (Phaseoulus vulgaris L.) y quichoncho (Cajanum cajan (L.) Mill.). Revista UDO Agrícola. 8(1): 61-66.

Quichimbo, P., Tenorio, G., Borja, P., Cárdenas, I., Crespo, P. y Célleri, R. 2012. Efecto sobre las propiedades físicas y químicas de los suelos por el cambio de la cobertura vegetal y uso del suelo: Páramo de Quimsacocha al sur del Ecuador. Suelos Ecuatoriales. 42(2): 138-153.

Sameen, R., Syed, W. y Alvina, G. 2016. Phytoremediation of saline soils for sustainable agricultural productivity. En: Plant metal interaction emerging remediation techniques. p. 465-468. Elsevier. Oxford.

Sánchez-Pérez, M.I., Muñoz-Mejía, C.Y., Quiroz-Velásquez, J.D., Mayek-Pérez, N. y Hernández-Mendoza, J.L. 2010. Cambios fisicoquímicos durante la germinación del maíz. Rev. Mex. Cienc. Agríc. 1(1): 89-93.

Sousa, E.A., Lemos-Filho, J.P. y Trombert, D.M. 2006. Imbibition of Swietenia macrophylla (meliaceae) Seeds: The Role of Stomata. Annals of Botany. 98: 213-217.

Zahedi, H., Shirani-Rad, A. H. y Tohidi-Moghadam, H. R. 2012. Zeolite and selenium application and their effects on production and physiological attributes of canola cultivars under water stress. Agrociencia. 46: 489-497. 